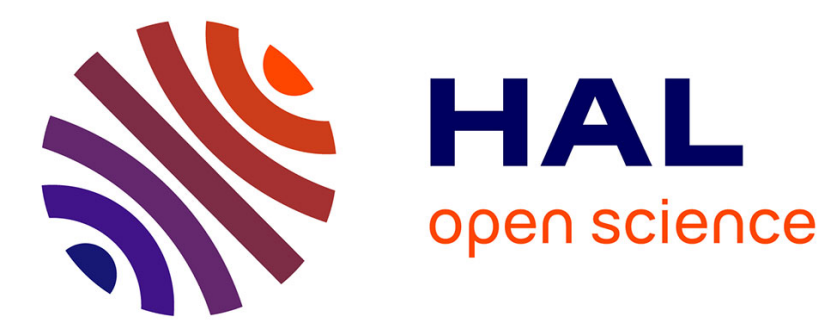

\title{
Numerical analysis of concrete creep on mesoscopic 3D specimens
}

B. Bary, C. Bourcier, T. Helfer

\section{To cite this version:}

B. Bary, C. Bourcier, T. Helfer. Numerical analysis of concrete creep on mesoscopic 3D specimens. CONCREEP 10 - 10th Conference on Mechanics and Physics of Creep, Shrinkage, and Durability of Concrete and Concrete Structuresth, Sep 2015, Vienne, Austria. cea-02509085

\section{HAL Id: cea-02509085 https://hal-cea.archives-ouvertes.fr/cea-02509085}

Submitted on 16 Mar 2020

HAL is a multi-disciplinary open access archive for the deposit and dissemination of scientific research documents, whether they are published or not. The documents may come from teaching and research institutions in France or abroad, or from public or private research centers.
L'archive ouverte pluridisciplinaire HAL, est destinée au dépôt et à la diffusion de documents scientifiques de niveau recherche, publiés ou non, émanant des établissements d'enseignement et de recherche français ou étrangers, des laboratoires publics ou privés. 


\title{
Numerical analysis of concrete creep on mesoscopic 3D specimens
}

\author{
B. Bary ${ }^{1}$, C. Bourcier ${ }^{2}$ and T. Helfer ${ }^{3}$
}

${ }^{1}$ CEA, DEN, DPC, SECR, Laboratoire d'Etude du Comportement des Bétons et des Argiles, Bât. 158, F-91191 Gif-sur-Yvette, France; email: benoit.bary@ cea.fr ${ }^{2}$ CEA, DEN, DM2S, Laboratoire de Génie Logiciel et de Simulation, Bât. 454, F91191 Gif/Yvette, France; email: christophe.bourcier@cea.fr

${ }^{3}$ CEA, DEN, DEC, SESC, Laboratoire de Simulation du comportement des Combustibles, Bât. 151, 13108 St Paul lez Durance, France; email: thomas.helfer@cea.fr

\begin{abstract}
In this paper we investigate analytically and numerically the creep behavior of concrete at mesoscale. The simulations are carried out with the finite element (FE) code $($ Cast3M) on 3D concrete specimens consisting in about 4600 polyhedral aggregates of various size and shapes randomly distributed in a box. Both matrix and Interfacial Transition Zone (ITZ) are considered as linear viscoelastic materials while the aggregates are elastic. Specific interface finite elements are introduced between the aggregates and the matrix to model the ITZ. The overall and intra-phase response of the numerical specimens when subjected to classical creep loadings is investigated, and then compared to the results of analytical estimations obtained with classical mean-field approximation schemes. These schemes are applied in the Laplace-Carson (LC) space, and the effects of the ITZ are accounted for via appropriate interface models. The results obtained for different ITZ thicknesses are analyzed so as to evaluate their respective influence on mortar and concrete materials.
\end{abstract}

\section{INTRODUCTION}

Two main functions are devoted to concrete structures in the nuclear context (containment building, waste storage structures...): containment and protection against radionuclide migration. The constitutive material must then meet high requirements in terms of performance and durability. In particular, loss of containment abilities due to long-term creep and induced development of cracks shall be prohibited. Concrete is a heterogeneous material made up at the mesoscale of linear elastic aggregates distributed in a mortar matrix whose behavior is timedependent. Besides, the presence of an ITZ between the aggregates and the matrix is known to also influence the overall behavior (see e.g. (Lavergne et al. 2015; Lutz et al. 1997; Scrivener et al. 2004)). It is then of particular importance to correctly characterize the respective role and impact of both phases and ITZ regarding the creep strains, since the initiation and propagation of cracks are strongly related to the local stresses and strains states as well as their history. 
In this paper we investigate analytically and numerically the creep behavior of concrete at mesoscale. The simulations are carried out on 3D concrete specimens generated by means of python scripts using the functionalities of the integration platform Salome (Bourcier et al. 2013; Salome). They consist in about 4600 polyhedral aggregates of various sizes and shapes obtained by a Voronoi space decomposition, and randomly distributed in a box. Only the coarser aggregates representing a volume fraction of $50 \%$ are considered in order to generate mesostructure meshes of reasonable size. Then, unstructured periodic meshes with tetrahedral elements are generated, and computations are carried out in the finite element code (Cast3M). The matrix and ITZ are considered as linear viscoelastic materials ruled by different generalized Maxwell models. Specific interface elements are introduced between the aggregates and the matrix to model the ITZ.

One purpose of the study is to analyze the overall and intra-phase response of the numerical specimens when subjected to classical creep loadings. In particular, the evolutions of averaged stresses and strains in the matrix and aggregate phases will be reported and compared to analytical estimations obtained with classical mean-field approximation schemes. These schemes will be applied in the Laplace-Carson (LC) space via the well-known elastic - linear viscoelastic correspondence principle. The effects of the ITZ will be accounted for via interfaces modelled with the linear-spring model (LSM) (see e.g. (Duan et al. 2007)) in the LC space. The influence of the ITZ thickness on the overall and local response of the specimens is studied. Further, an analysis regarding the evolutions of the mean stresses and strains in each aggregate and in matrix subvolumes is performed so as to quantify their fluctuations. Finally, the impact of the aggregate shape on both local and macroscopic response is analyzed through different mesostructures with flat and elongated particles.

\section{MESOSTRUCTURE GENERATION}

The procedure for constructing the numerical cubic samples is detailed in (Bourcier et al. 2013; de Larrard et al. 2013). The open-source python library Combs (Bourcier et al. 2013) based on the Computer-Aided Design code (Salome) is used to generate both geometry and meshes of the mesostructures. To improve the placement procedure time, the GJK 3D algorithm has been implemented in Combs for fast polyhedrons distance computation (see e.g. http://www.dyn4j.org/2010/04/gjkdistance-closest-points). The geometries considered in this paper are obtained by randomly distributing in a box polyhedral aggregates of various sizes and shapes obtained by a classical Voronoi space decomposition (de Larrard et al. 2013). To limit the FE computation time and the placement procedure, the choice of 4627 coarse aggregates has been retained, for a total volume fraction of 0.50 . Their number and volume are defined to match the ones of the corresponding spheres assembly according to the sieve curve described in (de Larrard et al. 2013). The total number of aggregates in the samples is greater than the prescribed one since the aggregates overlapping the surfaces of the mesostructures are cut and the remaining parts are placed on the opposite faces to ensure periodicity. To evaluate the effects of the aggregates shape, three mesostructures are generated, one with the initial Voronoi aggregates (denoted as 'isotropic' in the following), another with flat ones and the 
third with elongated inclusions. These flat and elongated aggregates are obtained by applying a scale transform of 3 on the initial aggregates along one or two perpendicular axes randomly defined. The Figure 1 presents a sample realization with isotropic (left) and flat (right) particles.
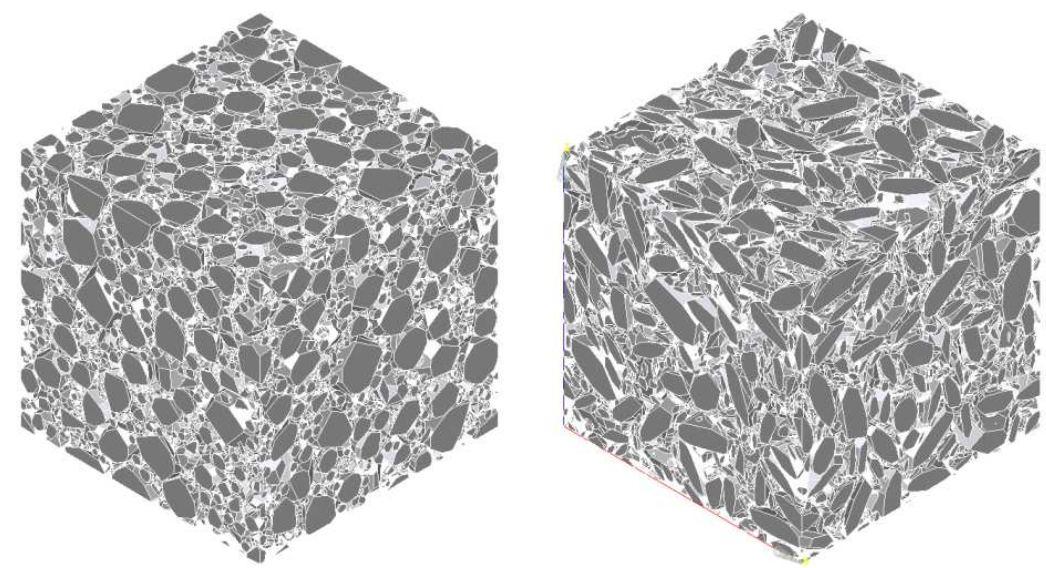

Figure 1. Sample with isotropic (left) and flat (right) aggregates.

For the numerical results to be comparable, the meshes are constrained to have a similar size. Here the number of linear tetrahedral finite elements ranges from 3.71 to $4.21 \times 10^{6}$.

\section{MODELLING}

As mentioned above, the behavior of the matrix material is assumed to be linear viscoelastic, with bulk $k^{m}(t)$ and shear moduli $\mu^{m}(t)$ ruled separately by a generalized Maxwell model with 4 elements (the element labeled as 0 is only composed of a spring) as:

$$
k^{m}(t)=k_{0}^{m}+\sum_{i=1}^{3} k_{i}^{m} e^{-t / \tau_{i}^{m}}, \mu^{m}(t)=\mu_{0}^{m}+\sum_{i=1}^{3} \mu_{i}^{m} e^{-t / \tau_{i}^{m}}
$$

in which $k_{i}^{m}$ and $\mu_{i}^{m}$ with $i \in\{0,3\}$ are the elastic moduli of the Maxwell elements, $\tau_{j}^{m}$ are the relaxation times. It is well-known that in the case of linear viscoelasticity the time-dependent problem may be equivalently reformulated as a linear elastic problem in the Laplace-Carson space (Mandel 1966), allowing to applying classical upscaling techniques. It is shown in (Thai et al. 2014) that in the particular case of the MT scheme and spherical particles, exact or semi-analytical solutions of the inversion problem can be derived. We choose here to apply this simplified methodology. The macroscopic behavior may then take the general form of a linear viscoelastic material:

$$
\boldsymbol{\sigma}(t)=3 \int_{0^{-}}^{t} k^{M T}(t-\tau) \frac{d \epsilon}{d \tau} d \tau \mathbf{1}+2 \int_{0^{-}}^{t} \mu^{M T}(t-\tau) \frac{d \boldsymbol{e}}{d \tau} d \tau
$$

with $\boldsymbol{\sigma}(t), \epsilon=1 / 3 \operatorname{tr}(\boldsymbol{\varepsilon})$ and $\boldsymbol{e}$ the macroscopic stress tensor, bulk and deviatoric part of the strain tensor $\varepsilon$, respectively; $k^{M T}$ and $\mu^{M T}$ are the homogenized bulk and 
shear moduli, which take the general form of Dirichlet series. The Young modulus of the aggregates is taken as $E=70 \mathrm{GPa}$ and its Poisson ratio as $v=0.3$. The parameters of the Maxwell models are identified on a classical concrete creep test realized by (Ladaoui 2010) (see (Bary et al. 2014)). To simplify, the relaxation times are chosen a priori, in accordance with the duration of the creep tests (300 days). The behavior of the interface between the aggregates and the matrix is also assumed to be ruled by generalized Maxwell models. As in concrete the mechanical properties of the ITZ are known to be more compliant than the ones of the mortar matrix and aggregates, we chose to apply the LSM in the LC space to simulate the interface effects. The interface conditions for the LSM are (e.g., (Duan et al. 2007; Hashin 1991)):

$$
[\widetilde{\boldsymbol{\sigma}}] \cdot \boldsymbol{n}=0, \tilde{\mathbf{k}} \cdot[\boldsymbol{u}]=\widetilde{\boldsymbol{\sigma}} \cdot \boldsymbol{n}
$$

where $\boldsymbol{n}, \tilde{\mathbf{k}}, \boldsymbol{u}$ are the unit normal vector to the interface, the second order tensor characterizing the elastic parameters in the LC space (the 'tilde' notation designates the properties expressed in the LC space), and the displacement; [-] denotes the jump of the corresponding quantities. In the case where the interface is thin and compliant with respect to the inclusion, i.e. $h \ll R_{I}, E_{c} \ll E_{I}$ and $\mu_{c} \ll \mu_{I}$ with $h$ the thickness of the interphase, $R_{I}$ the radius of the spherical inclusion, $E_{c}$ and $\mu_{c}$ the Young and shear moduli of the interphase, $E_{I}$ and $\mu_{I}$ the Young and shear moduli of the inclusion, the tensor $\tilde{\mathbf{k}}$ can be expressed as $\tilde{\mathbf{k}}_{\mathbf{n}}=\tilde{\mathrm{k}}_{n} \boldsymbol{n} \otimes \boldsymbol{n}+\tilde{\mathrm{k}}_{t} \boldsymbol{s} \otimes \boldsymbol{s}+\tilde{\mathrm{k}}_{t} \boldsymbol{t} \otimes \boldsymbol{t}$ with

$$
\tilde{\mathrm{k}}_{n}=\frac{2 \tilde{\mu}_{c}\left(1-\tilde{v}_{c}\right)}{h\left(1-2 \tilde{v}_{c}\right)}, \tilde{\mathrm{k}}_{t}=\frac{\tilde{\mu}_{c}}{h}
$$

in which $\boldsymbol{s}$ and $\boldsymbol{t}$ are the two orthogonal unit vectors in the tangent plane of the interface and $\tilde{v}_{c}$ is the Poisson ratio of the interphase. In the following, we will retain this model for characterizing the viscoelastic properties of the interfaces in the FE simulations, providing $\mathrm{k}_{n}$ and $\mathrm{k}_{t}$ are expressed in the time space. Further, we propose to estimate the overall properties of the heterogeneous material by making use and extending the approach due to (Duan et al. 2007), based on a replacement procedure. In this approach, the elastic composite inclusions composed of the aggregates and their surrounding interfaces are replaced by equivalent homogeneous spherical inclusions. The expressions of the moduli for these equivalent particles depend then on both inclusion and interphase properties; as they are lengthy, they are not recalled here, see (Duan et al. 2007) for details. Since these formulas are established in an elastic framework, they are applied here in the LC space. Once the composite inclusions are substituted by equivalent homogeneous ones, the MT scheme may be applied to obtain the overall properties of the concrete material.

\section{SIMULATION RESULTS}

We propose to perform the simulations in the $\mathrm{FE}$ code Cast $3 \mathrm{M}$ with the different following values of interphase thickness $h=1,10,50,100$ and $250 \mu \mathrm{m}$. A calculation with no interfaces between aggregates and matrix is also carried out to control the accuracy of the results with smaller thicknesses. Note that the higher values of thickness considered are unrealistic for concrete, but are representative for mortars, provided the aggregates are regarded as sand particles. The loading corresponds to a typical creep test, defined by a constant normal stress 
of $-25.8 \times 10^{6} \mathrm{~Pa}$ applied on the sample boundaries in the direction 1 and zero stress on the other faces, via uniform stresses boundary conditions (BC). Such BC are preferred as they give results close to the periodic ones, for a much lower computation time (Bary et al. 2014). The computations are performed on standard Linux machine with 20 cores and 128 Go RAM. The total simulated time is 300 days, reached in 32 time steps. The computation time is about 10-12 hours, and additional 10-12 hours are needed for post-processing the results (i.e. calculating the average stresses and strains in all inclusions and matrix subvolumes for all time steps). The mechanical properties of the interphase are supposed to be half those of the matrix (e.g. (Neubauer et al. 1996)). The mechanical models for both matrix and interphase materials have been implemented via the Mfront code generator developed at CEA (MFront).

Figure 2 presents the evolutions of the creep longitudinal and transversal strains obtained numerically by FEM (symbols) and analytically (lines) for the different interface thicknesses and the isotropic aggregate case. We observe as expected that the magnitude of strains progressively increases for higher values of thickness. The differences are negligible between $h=1$ and $10 \mu \mathrm{m}$, and are about 6,14 and $33 \%$ between $h=1$ and 50,100 and $250 \mu \mathrm{m}$, respectively. Note that the results with no interface are omitted as they are superposed with the $h=1$ thickness ones. One may conclude that the presence of ITZ, whose typical thickness is around $20-25 \mu \mathrm{m}$ (Scrivener et al. 2004) in concrete at mesoscale (i.e. considering the matrix as mortar), is not significant regarding the estimation of macroscopic response of the material. By contrast, for mortars which correspond approximately to the case of a thickness of $250 \mu \mathrm{m}$ with respect to the aggregate sizes, they appear very influential and should not be neglected. Note that all the results shown here may be somewhat moderated since ITZ are modelled as idealized interfaces with very low mechanical properties, which may not be totally realistic.

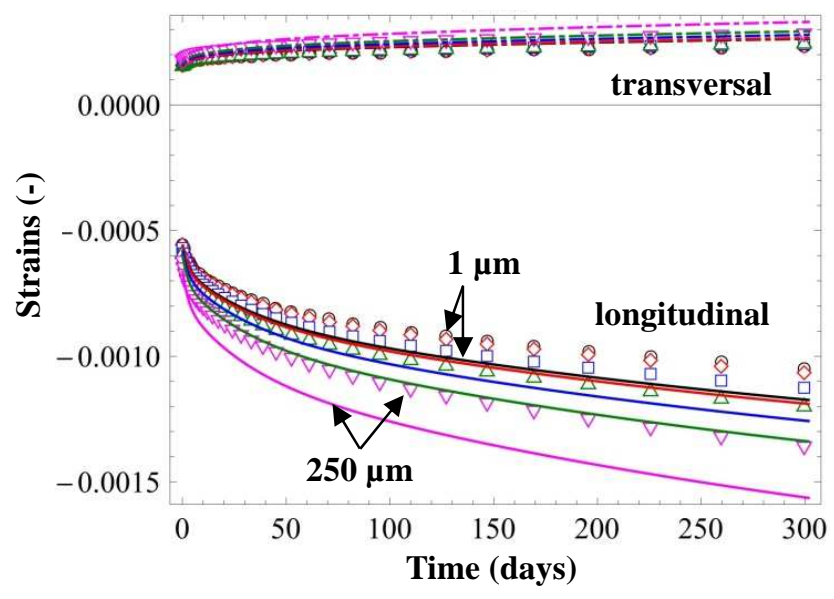

Figure 2. Longitudinal and transversal creep strains obtained numerically (symbols) and analytically (lines) for interface thicknesses of 1, 10, 50, 100 and $250 \mu \mathrm{m}$. 
We also observe that the strains estimated by the model overestimate in magnitude the numerical ones for all interface thicknesses. Several causes may explain these discrepancies: one is due to the inherent use of the MT scheme whose application domain should not in principle exceed about $30 \%$ of inclusion volume fraction; another lies in the inclusion shape considered, spherical for the model and polyhedrons for the FE. Biases introduced by the insufficiently fine meshes and the considered BC are also well-known sources of inaccuracies. Figure 3 shows the evolutions of average stresses (left) and strains (right) in the aggregates and matrix in the direction of creep loading, obtained numerically (symbols) and analytically (lines) for the different interface thicknesses. It should be noted that the average strains in the aggregate phase (contrary to the average stresses in the case of LSM) cannot be directly obtained via the estimation of the strain concentration tensor since in the approach of (Duan et al. 2007) the MT scheme is applied to the material exhibiting equivalent homogeneous inclusions in replacement of the composite sphere-interface ones. This Figure indicates that an increase of the interface thickness tends to reduce the magnitude of the stress in the aggregates and to augment it in the matrix; this is the reverse for the strain: it increases in the matrix and decreases in the aggregates. Again, the model provides estimations relatively close to the FE simulations.

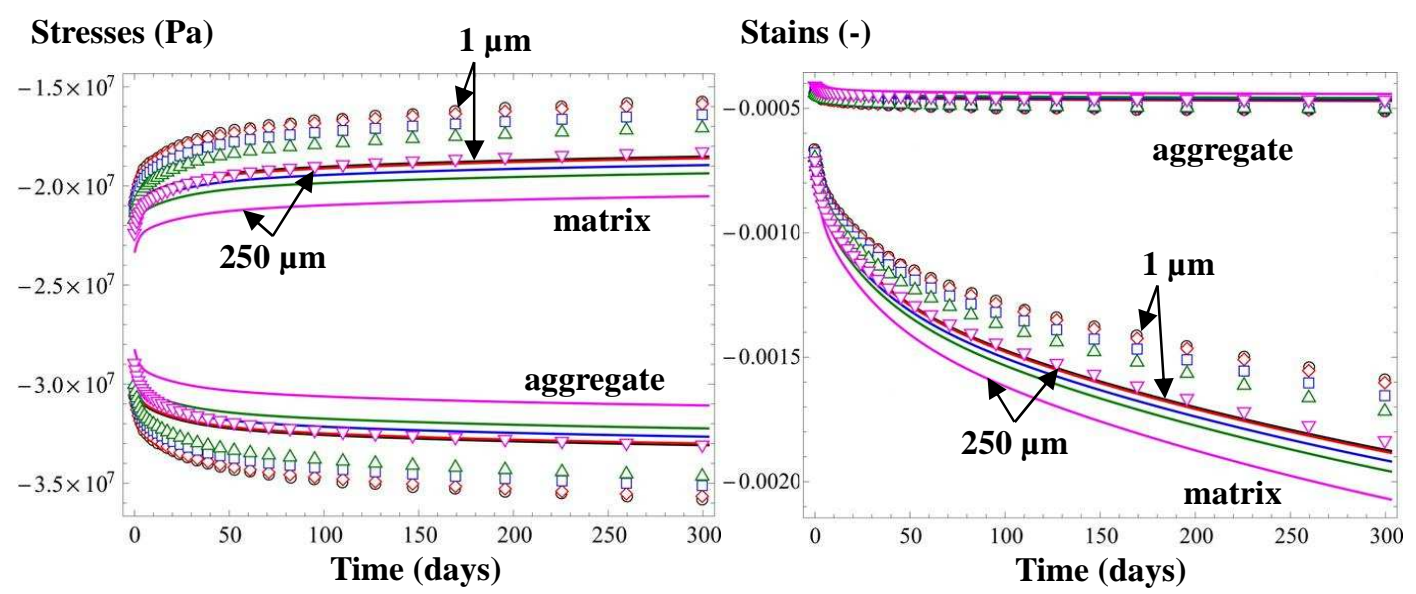

Figure 3. Average stresses (left) and strains (right) in both aggregate and matrix phase, obtained numerically (symbols) and analytically (lines) for different interface thicknesses ranging from 1 to $250 \mu \mathrm{m}$.

Figure 4 presents the probability distribution functions (PDF) of the longitudinal average stress in the aggregates and matrix subvolumes for the interface thicknesses of 1 (left) and 250 (right) $\mu \mathrm{m}$, and at 0.38, 24.5 and 300 days. The matrix subvolumes are obtained by dividing the matrix with a voxel-type procedure. Here the dimension of a voxel is chosen as $1 / 20$ of the edge dimension of the initial box (i.e. 8000 subvolumes are expected). We observe that the stress in the aggregates is more dispersed than in the matrix subvolumes, in particular in the case of the smaller interface thickness. This dispersion tends to reduce for higher thickness (Figure 4 right). Moreover, in all cases the PDF are more dispersed at later ages. These results indicate that the presence of interfaces affects notably the mean stress in both matrix 
and aggregate phases, but also the dispersion essentially in the inclusive phase. Besides, the relatively high dispersion proves that important stress concentrations arise locally, meaning that microcracking is likely to occur in particular in the matrix and ITZ phases, whose strength is in general weaker than in the aggregate one.
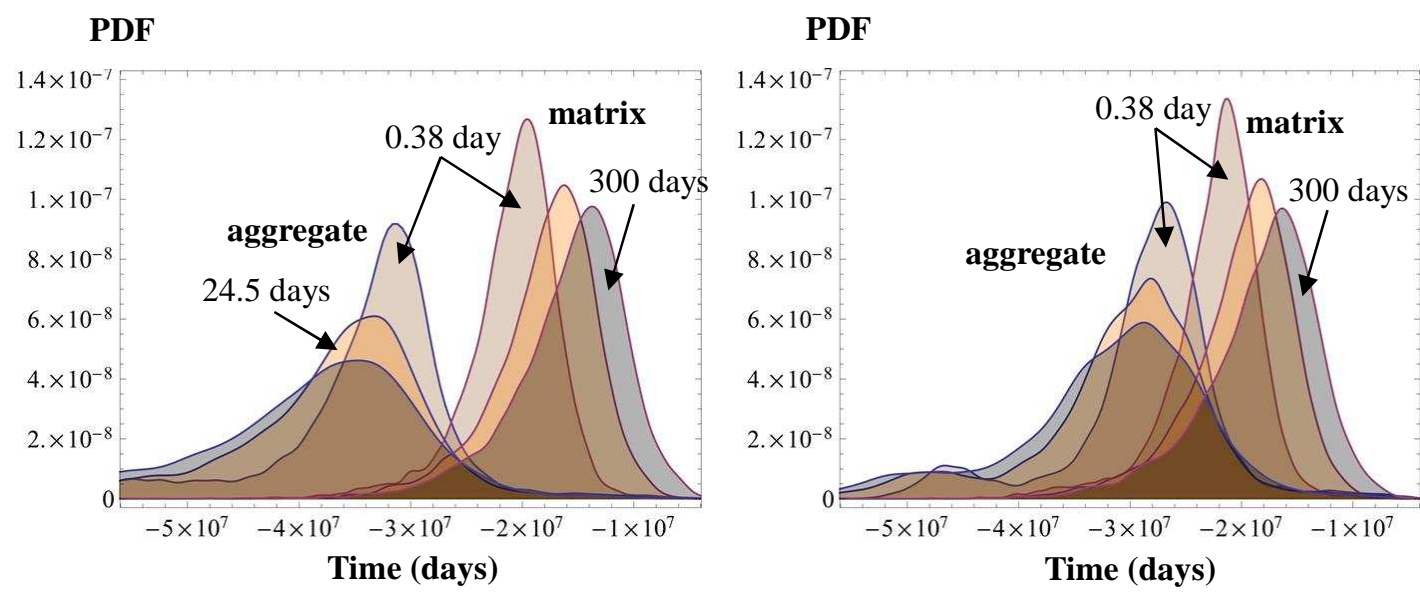

Figure 4. FE Probability Distribution Function for the average longitudinal strain in both matrix subvolumes and aggregates for the $1 \mu \mathrm{m}$ (left) and $250 \mu \mathrm{m}$ (right) interface thickness and at $0.38,24.5$ and 300 days.

Finally, we compare on Figure 5 the longitudinal creep strain obtained in the cases of isotropic, flat and elongated aggregates, and for the interface thickness of 1 , 100 and $250 \mu \mathrm{m}$. The curve obtained for a mesostructure containing 1052 spherical particles representing a volume fraction of $50 \%$ is also depicted. We note that the effects of the aggregate shape as considered here (i.e. polyhedrons with different aspect ratios) are not important regarding the macroscopic creep response, whatever the interface thicknesses. This aspect is in agreement with results reported in e.g. (Lavergne et al. 2015).

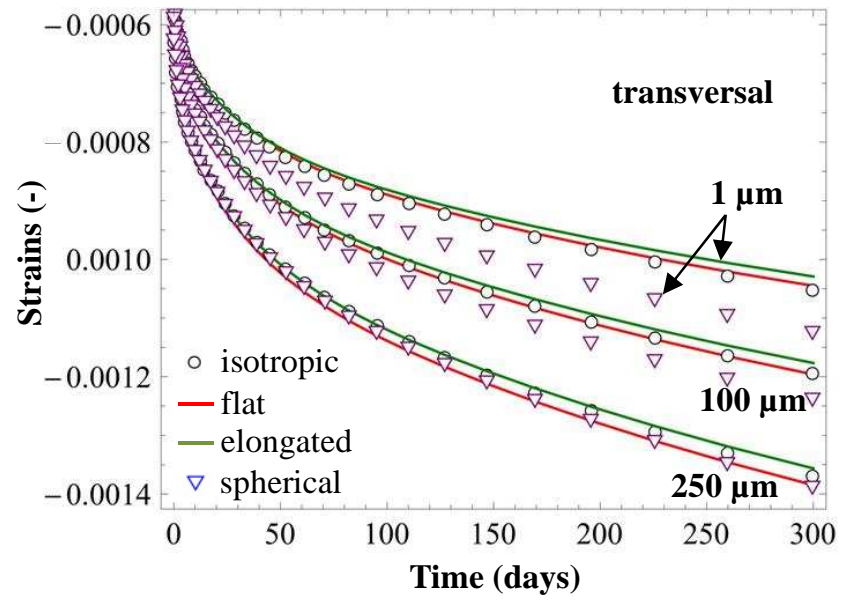

Figure 5. Longitudinal creep strain obtained numerically for different aggregate shapes and 3 interface thicknesses. 
By contrast, significant discrepancies are observed between spherical and nonspherical aggregates for lower ITZ thickness, which tend to reduce and even cancel for higher ones. However, it should be noted that the mesostructure with spherical particles has much less inclusions and then interfaces than the ones with polyhedrons. This was imposed to obtain meshes of similar size, due to the fact that curved surfaces necessitate a much finer discretization than plane ones. Consequently, the results may only be compared cautiously in particular for high ITZ thicknesses.

\section{CONCLUSION}

In this paper, we have analyzed analytically and numerically the viscoelastic behavior of concrete at mesoscale. FE simulations have been carried out on 3D concrete specimens with 4600 polyhedral aggregates randomly distributed in a box and representing a volume fraction of 50\%. Both matrix and ITZ behaviors have been considered as linear viscoelastic and the aggregates one as elastic. Specific FE interface elements have been introduced to model the ITZ, assumed to be more compliant than the matrix. The overall and intra-phase responses in the numerical specimens have then been investigated when subjected to classical creep loading, and compared to the analytical estimations obtained with classical mean-field approximation schemes accounting explicitly for ITZ and applied in the LC space. The results obtained show that typical ITZ thicknesses for concrete of 20-25 $\mu \mathrm{m}$ do not affect significantly the macroscopic and intra-phase response, contrary to the case of mortars whose behavior is comparatively notably influenced. As expected, increasing the ITZ thickness leads globally to a rise of the creep strains magnitude, i.e. the material becomes more compliant. Moreover, it implies a reduction (in magnitude) of the stresses in the aggregates and an increase of both stresses and strains in the matrix phase. A significant dispersion has been observed in the average creep stress per aggregate and per matrix subvolumes, indicating potentially important local stress concentrations. Higher ITZ thicknesses tend to slightly reduce this dispersion. The effects of the aggregate shape (i.e. flat or elongated polyhedrons with aspect ratio of 3) are weak on the macroscopic response, whatever the interface thickness. More important discrepancies are observed with spherical particles.

Future works will focus on the introduction of a more realistic ITZ behavior discarding the hypothesis of very low mechanical properties. Coupling with damage will also be a challenging aspect to study both analytically and numerically.

\section{REFERENCES}

Bary, B., Gélébart, L., Adam, E., and Bourcier, C. (2014). "Numerical analysis of linear viscoelastic 3D concrete specimens: Comparison between FE and FFT methods." Computational Modelling of Concrete Structures, St. Anton am Arlberg, Austria, 373-381.

Bourcier, C., Dridi, W., Chomat, L., Laucoin, E., Bary, B., and Adam, E. (2013). "Combs: open source python library for RVE generation. Application to microscale diffusion simulations in cementitious materials." Paris. 
Cast3M. www-cast3m.cea.fr.

Duan, H. L., Yi, X., Huang, Z. P., and Wang, J. (2007). "A unified scheme for prediction of effective moduli of multiphase composites with interface effects: Part II-Application and scaling laws." Mechanics of Materials, 39(1), 94103.

Hashin, Z. (1991). "Thermoelastic properties of particulate composites with imperfect interface." Journal of the Mechanics and Physics of Solids, 39(6), 745-762.

Ladaoui, W. (2010). "Etude expérimentale du comportement Thermo-HydroMécanique à long terme des BHP destinés aux ouvrages de stockage des déchets radioactifs." Université de Toulouse, Université Toulouse III-Paul Sabatier.

De Larrard, T., Bary, B., Adam, E., and Kloss, F. (2013). "Influence of aggregate shapes on drying and carbonation phenomena in 3D concrete numerical samples." Computational Materials Science, 72, 1-14.

Lavergne, F., Sab, K., Sanahuja, J., Bornert, M., and Toulemonde, C. (2015). "Investigation of the effect of aggregates' morphology on concrete creep properties by numerical simulations." Cement and Concrete Research, 71, 1428.

Lutz, M. P., Monteiro, P. J. ., and Zimmerman, R. W. (1997). "Inhomogeneous interfacial transition zone model for the bulk modulus of mortar." Cement and Concrete Research, 27(7), 1113-1122.

Mandel, J. (1966). Cours de mécanique des milieux continus. Gauthier-Villars.

MFront. www.tfel.sourceforge.net.

Neubauer, C. M., Jennings, H. M., and Garboczi, E. J. (1996). "A three-phase model of the elastic and shrinkage properties of mortar." Advanced Cement Based Materials, 4(1), 6-20.

Salome. www.salome-platform.org.

Scrivener, K. L., Crumbie, A. K., and Laugesen, P. (2004). "The interfacial transition zone (ITZ) between cement paste and aggregate in concrete." Interface Science, 12(4), 411-421.

Thai, M.-Q., Bary, B., and He, Q.-C. (2014). "A homogenization-enriched viscodamage model for cement-based material creep." Engineering Fracture Mechanics, 126, 54-72. 